\title{
A survey on Block Truncation Coding based Content Based Image Retrieval
}

\author{
R.Sahaya Jeya Sutha ${ }^{\# 1}$, Dr.D.S.Mahendran ${ }^{\# 2}$, Dr.S.John Peter $^{\# 3}$ \\ ${ }^{\# 1}$ Research Scholar, Manonmaniam Sundaranar University, Tirunelveli, India \\ \#2 Asso. Professor, Dept. of Computer Science, Aditanar College of Arts \& Science, Tiruchendur, India \\ \#3 Asso. Professor \& Head, Dept. of Computer Science, St.Xavier's College, Palayamkottai, India
}

\begin{abstract}
Content Based Image Retrieval (CBIR) is an important active research topic helps the user to search the relevant images from the large image collections by analyzing the content of the user required query image. With the explosive growth of image databases over internet and the availability of image capturing devices like mobile phones, digital camera and scanners, efficient image retrieval techniques are required. Many researchers proposed various techniques to improve the retrieval accuracy of content based image retrieval in a reasonable amount of time. When compared to other image compression techniques, the Block truncation coding (BTC) has been considered as a very efficient compression technique which requires least computational complexity and it can also effectively employed to index images in database for CBIR applications. This paper attempts to provide a comprehensive survey on various image retrieval techniques using BTC compression based indexing techniques. Further, some other issues, retrieval performance metrics and future enhancements are also discussed.
\end{abstract}

Keywords

Content Based Image Retrieval (CBIR), Block Truncation Coding (BTC), Error Diffusion BTC (EDBTC), Ordered Dither BTC (ODBTC), Dot Diffusion BTC (DDBTC).

\section{INTRODUCTION}

With the development of internet and the inventions of efficient image capturing devices like mobile phones, high resolution digital cameras and scanners, cheap storage, the volume of digital images is increasing exponentially. This created a need of efficient image retrieval techniques for users in various field.

There are two types of image retrieval techniques are: Text Based Image Retrieval (TBIR) and Content Based Image Retrieval (CBIR) [20]. In text based approach, the images are manually annotated by text descriptors, which are then used by a database management system to perform image retrieval. It requires a considerable level of human labor for manual annotation. But manual work is an expensive task for large image databases, and is often subjective, contextsensitive and incomplete. To overcome the above drawbacks CBIR was introduced. CBIR is the application of computer vision which is also known as query by image content (QBIC) and content based visual information retrieval (CBVIR).

Content based image retrieval (CBIR) is a method to search the relevant images from the large image collections by analyzing the content of the user required query image [20][31]. The main advantage of CBIR is that the user is not required to provide an explicit description of the target, which is instead automatically computed by the system.

The CBIR systems operate in Pixel and Compressed domain. In the pixel domain approach for feature extraction first we need to decompress the image. But in the compressed domain approach image retrieval can be achieved directly from the compressed image without decompressing it.

Today's CBIR systems suffer from main problems like unsatisfactory results and the long response time, because of the inaccurate existing methods and large memory required for storing a large scale image dataset. CBIR techniques based on the compressed data stream have become an important issue to rectify these problems. In this scenario, the feature extractor simply generates an image feature from compressed data stream without performing the decoding process. 
Compressed domain CBIR is classified into DCT, Wavelet, and Vector Quantization (VQ) based. JPEG and BTC are some of the famous compressed domain methods of DCT and VQ based methods. The BTC based image retrieval is suitable for image retrieval requiring fast execution since their simplicity and low computational burden.

The attractive properties of BTC compression led to its deployment in several applications, including image watermarking, data hiding, image restoration, video retrieval and content based image retrieval. The benefit of BTC based CBIR is that the feature vector of image is independent of the image dimensions (no need of all database images to be of same size to that of query image).

This paper surveys the various BTC compression based CBIR system along with the suitable technologies involved in each step of CBIR.

\section{SYSTEM ARCHITECTURE OF TYPICAL CBIR}

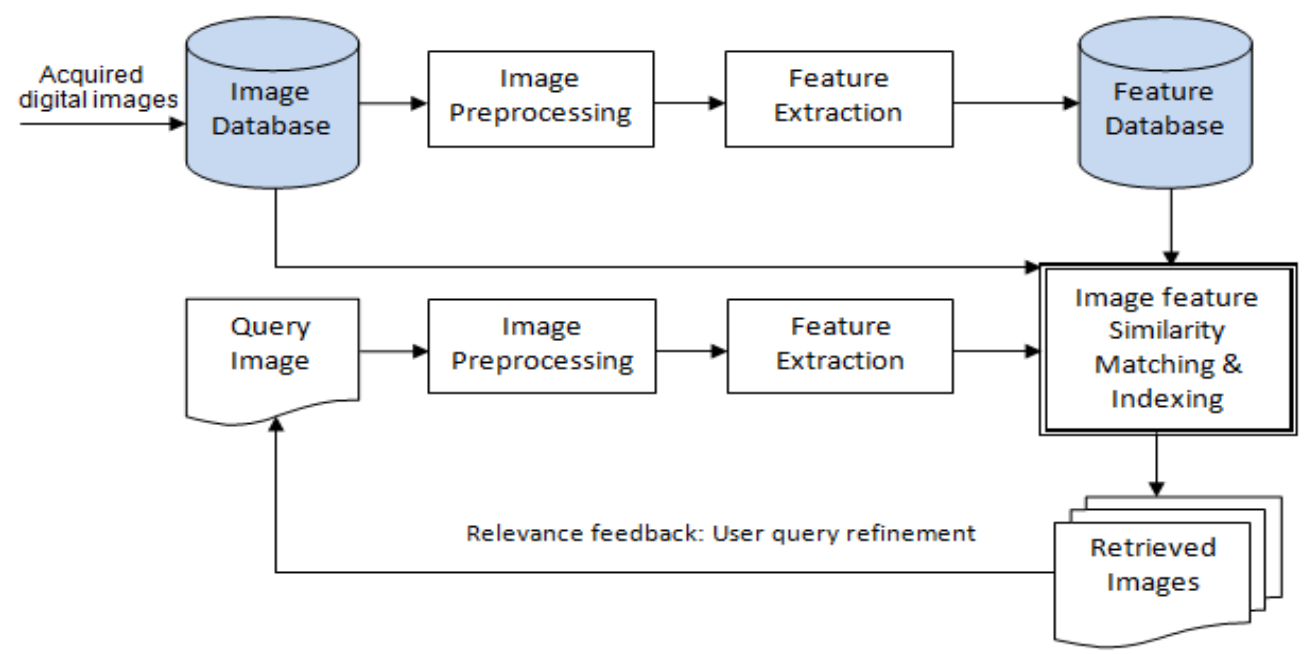

Fig 1. Block diagram of the typical CBIR system

The basic steps of typical CBIR system [16][20][31] are shown in Fig 1. Before we discuss related works done using BTC based CBIR methods, let us briefly discuss the different feature extraction, similarity measurements and performance measurements relevant with these BTC based CBIR.

\section{FEATURE EXTRACTION FOR CBIR}

The most common features are based on color, texture, and shape also known as low level features. It can be accurately and automatically extracted from the image. Single feature based retrieval systems were not giving satisfactory results because image contains several visual features. So that different combinations of the visual features are used to retrieve the required image.

In CBIR, the images are automatically indexed by summarizing their visual contents from the extracted low-level features. However, extracting all visual features of an image is a difficult task and there is a problem called semantic gap. Image grouping into semantically meaningful categories based on low-level visual features very hard. In order to alleviate these limitations, some researchers use both low level and high level techniques together using different features.

For the low-level features, only some key information is extracted from the images. The former works, [3],[12]-[14] and [27]-[28], mainly utilized the compressed stream such as BTC, JPEG and DCT to assemble the features.

Some of the halftoning-based BTC compressions (EDBTC, ODBTC, DDBTC) are having good image quality, to extract the important features for efficient image retrieval. The low-level features, e.g., texture and color, are constructed by VQ-indexed histogram from BTC bitmap, maximum, and minimum quantizers. 


\subsection{BTC compression based features}

The BTC is not only a good capability for image compression but also offers an effective way to index images for the CBIR system. The BTC based compressions decomposes an image into a bitmap image and two color quantizers which are subsequently exploited for deriving the image feature descriptor. The feature derived from bitmap image characterizes the statistics of the spatial patterns of the blocks. The other feature derived from two color quantizers characterizes the statistics of the color correlation within the blocks.

In Ref. [3], Guoping Qiu proposed Block Color Co-occurrence Matrix (BCCM) and Block Pattern Histogram $(\mathrm{BPH})$ to derive two image content description features from the two color quantizers and the bitmap image respectively using the BTC compressed stream.

In Ref. [12], J. M. Guo et.al, introduced Color Co-occurrence Feature (CCF) and Bit Pattern Feature (BPF) image features to characterize the image contents using the ODBTC compressed stream. The $\mathrm{CCF}$ is derived from the two color quantizers, and the BPF is from the bitmap image.

In Ref. [13], J. M. Guo et.al, introduced Color Histogram Feature (CHF) and Bit Pattern Histogram Feature (BHF) image features to characterize the image contents using the EDBTC compressed stream. The CHF is derived from the two color quantizers, and the BHF is from the bitmap image. In Ref. [14], J. M. Guo et.al, proposed Color Histogram Feature (CHF) and Bit Pattern Feature (BPF) image features to characterize the image contents using the DDBTC compressed stream. The CHF and $\mathrm{BPF}$ are derived from the two color quantizers, bitmap image respectively.

\subsubsection{BTC or Block Color Co-occurrence Matrix (BCCM)}

From [3], the BCCM of an image is defined as the probability of the co-occurrence of two mean colors within the BTC blocks. Each color mean value quantize J-color and it makes the hash table. Formally

$$
\operatorname{BCCM}(i, j)=P_{r}\left(\operatorname{IHash}\left\{I_{1}\right\}=i \mid \operatorname{IHash}\left\{I_{2}\right\}=j\right), \forall i, j \in[0, J-1]
$$

Where $I_{1}$ and $I_{2}$ are the pair of local color indices of the BTC mean colors within the same block. IHash be a color indices hash table which has the local color indices. Equivalently, BCCM(i, j) gives the probability that a $\mathrm{BTC}$ coded block having one mean color $\mathrm{MC}_{1}$ (local color quantized color) being mapped to the $i^{\text {th }}$ color in the global color table and other mean color $\mathrm{MC}_{2}$ (local color quantized color) being mapped to the $j^{\text {th }}$ color in the global color table.

\subsubsection{Block Pattern Histogram (BPH)}

From [3], another measure BPH used for characterizing the image content. First, create a codebook of binary patterns for the bitmap of BTC code using binary vector quantization. Let $Q=$ $\left\{Q_{1}, Q_{2}, \ldots Q_{N}\right\}$ be the bitmap codebook consists of $N$ codewords and $Q_{i}$ is a binary pattern. Let $B=\{b(i, j), i=1,2, \ldots, m, j=1,2, \ldots, n\}$ be the bitmap of the BTC code, the pattern index of the bitmap, $\mathrm{P}_{\mathrm{I}}$, is the index of the bitmap codeword that is most similar to $\mathrm{B}$, formally defined as

$$
P_{I}=\arg \left({ }_{\forall j}^{\min }\left\{D H\left(B, Q_{j}\right)\right\}\right)
$$

where $\mathrm{DH}\left(\mathrm{B}, \mathrm{Q}_{\mathrm{j}}\right)$ denotes the Hamming distance between the two binary patterns (vectors). The BPH is defined as

$$
\mathrm{BPH}(\mathrm{i})=\mathrm{P}_{\mathrm{r}}\left(\mathrm{P}_{\mathrm{I}}=\mathrm{i}\right), \mathrm{i}=1,2, \ldots, \mathrm{N}
$$

$\mathrm{BPH}(\mathrm{i})$ is the probability that a BTC bitmap is mapped to codeword $\mathrm{Q}_{\mathrm{i}}$ in the bitmap codebook.

\subsubsection{Color Co-Occurrence Feature (CCF)}

The minimum and maximum color quantizers are firstly indexed using a specific color code book. Using these indexed values color co-occurrence matrix is constructed. Then CCF is derived from the color co-occurrence matrix at the end of computation. LBG Vector Quantization (LBGVQ) generates a representative codebook from a number of training vectors. The Fig. 2 shows the block diagram of the CCF computation. 


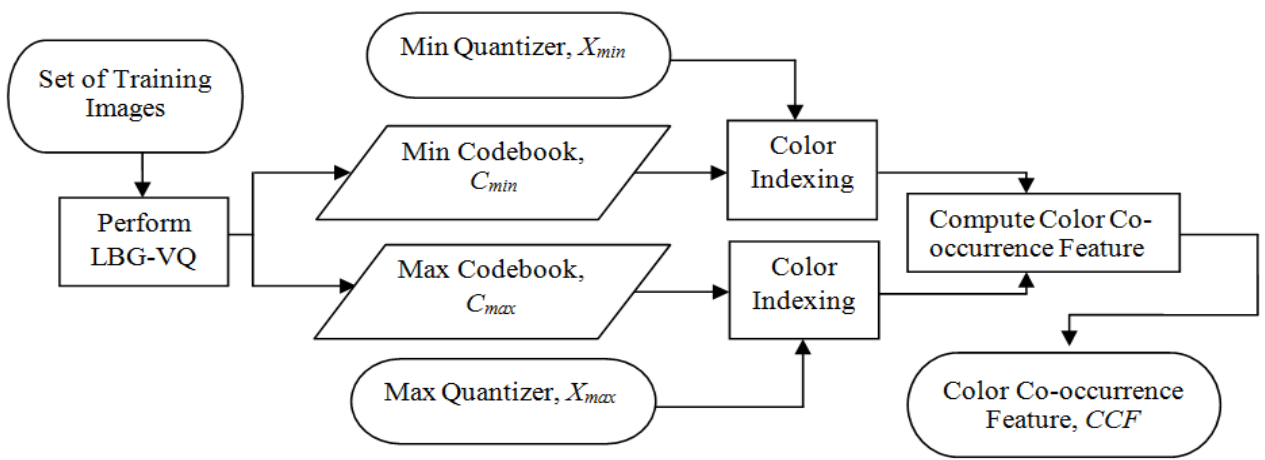

Fig 2. Block diagram for computing the color co-occurrence feature

\subsubsection{Bit Pattern Feature (BPF)}

Bit Pattern Feature (BPF), characterizes the image edges, shapes and contents. The BPF is simply derived as the occurrence probability of the bitmap image mapped into the specific bit pattern codeword. The binary LBG vector quantization produces a representative bit pattern codebook from a set of training bitmap images obtained from the ODBTC encoding process. At the codebook generation stage, all code vector components may have intermediate real values between zero (black pixel) and one (white pixel) as opposed to binary values. At the end of training stage, the hard thresholding performs the binarization of all code vectors to yield the final result. Similar to that of the $\mathrm{CCF}$, the BPF only needs a simple computation, making it suitable for real applications where fast response is required. The Fig. 3 shows the block diagram for deriving the BPF.

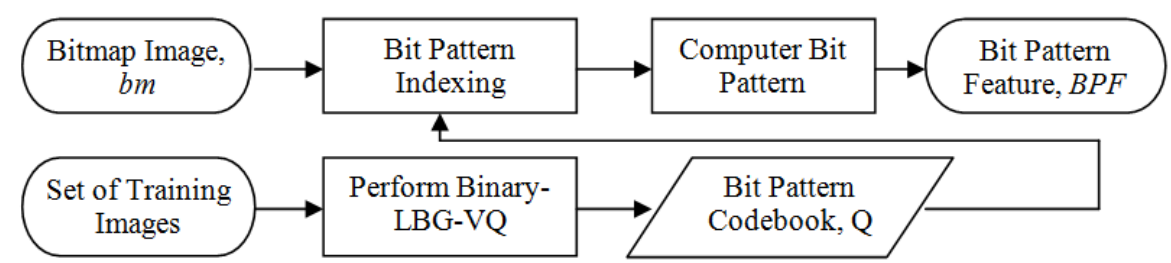

Fig 3. Block diagram for computing the Bit Pattern Feature (BPF)

\subsubsection{Color Histogram Feature (CHF)}

The CHF is simply derived from the two EDBTC color quantizers. In this method, the $C H F_{\min }$ and $\mathrm{CHF}_{\max }$ are developed from the color minimum and maximum quantizers, respectively for capturing color information from a given image. These features represent the combination of pixel brightness and color distribution in an image. It only calculates the occurrence of a certain color of codebook appeared in an image. The Fig. 4 shows the block diagram of CHF computation.

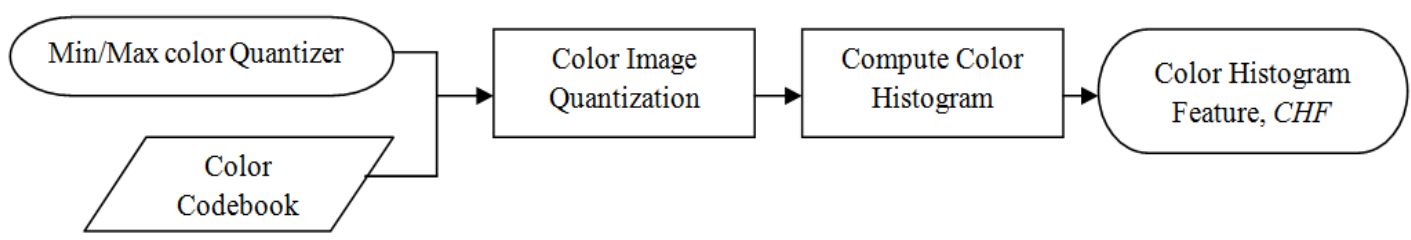

Fig 4. Block diagram of the CHF Computation

\subsubsection{Bit Pattern Histogram Feature (BHF)}

The BHF feature captures the visual pattern, edge and textural information in an image. This can be obtained by tabulating the occurrence of a specific bit pattern codebook in an image. The Fig. 5 shows the BHF computation. The BHF can be viewed as the histogram of the indexed bit pattern of the EDBTC bitmap image. 


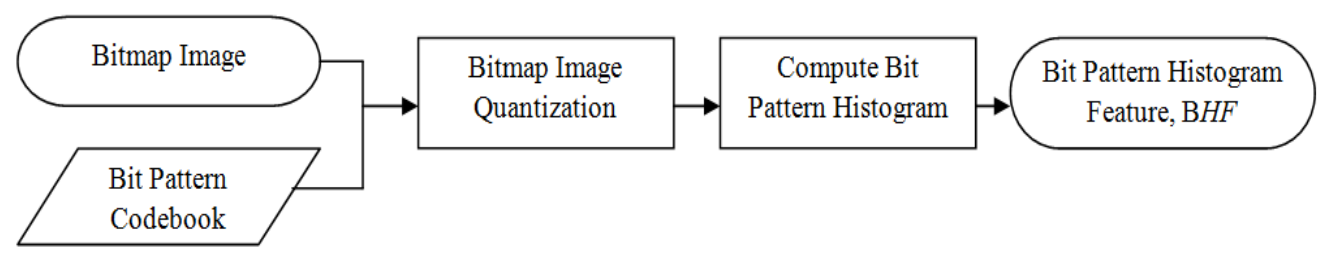

Fig 5. Block diagram of the BHF Computation

\section{SIMILARITY MEASUREMENT FOR CBIR}

The similarity between two images of a query image and the set of images in the database as target image can be measured using the distance measure like $\mathrm{L}_{1}, \mathrm{~L}_{2}, \ldots, \mathrm{L}_{\mathrm{n}}$ distances, $F u$ distance, modified Canberra distance, etc. The distance plays the most important role in the CBIR system since the retrieval result is very sensitive with the chosen distance metric. The similar images to the query image is returned and ordered based on their similarity distance score. The lowest score indicates the most similar image to the query image.

The similarity distances between the two images $\delta$ (query,target) can be formally defined under various distance metrics as follows:

\section{1 $L_{1}$ distance}

$$
\begin{gathered}
\alpha_{1} \sum_{k=1}^{N_{\text {min }}}\left|C H F_{\text {min }}^{\text {query }}(k)-C H F_{\text {min }}^{\text {target }}(k)\right|+\alpha_{2} \sum_{k=1}^{N_{\text {max }}}\left|C H F_{\text {max }}^{\text {query }}(k)-C H F_{\text {max }}^{\text {target }}(k)\right| \\
+\alpha_{3} \sum_{k=1}^{N_{b}}\left|B P F^{q u e r y}(k)-B P F^{\text {target }}(k)\right|
\end{gathered}
$$

\section{2 $L_{2}$ distance}

$$
\begin{gathered}
\alpha_{1} \sum_{k=1}^{N_{\min }}\left(C H F_{\min }^{\text {query }}(k)-C H F_{\text {min }}^{\text {target }}(k)\right)^{2}+\alpha_{2} \sum_{k=1}^{N_{\max }}\left(C H F_{\text {max }}^{\text {query }}(k)-C H F_{\text {max }}^{\text {target }}(k)\right)^{2} \\
+\alpha_{3} \sum_{k=1}^{N_{b}}\left(B P F^{\text {query }}(k)-B P F^{\text {target }}(k)\right)^{2}
\end{gathered}
$$

\section{$4.3 \chi^{2}$ distance}

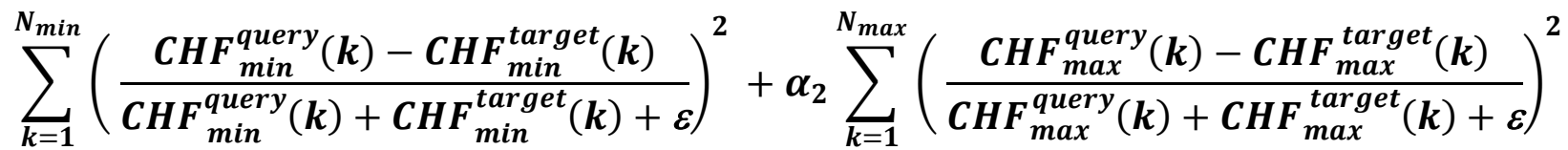

$$
\begin{aligned}
& +\alpha_{3} \sum_{k=1}^{N_{b}}\left(\frac{\text { BPF }^{\text {query }}(k)-\text { BPF }^{\text {target }}(k)}{\text { BPF query }_{(k)+\text { BPF }^{\text {target }}(k)+\varepsilon}}\right)^{2}
\end{aligned}
$$

\subsection{Fu distance}

$$
\frac{L_{2} \text { distance }}{\left|C H F_{\text {min }}\right|+\left|C H F_{\text {max }}\right|+|B P F|}
$$




\subsection{Modified Canberra distance}

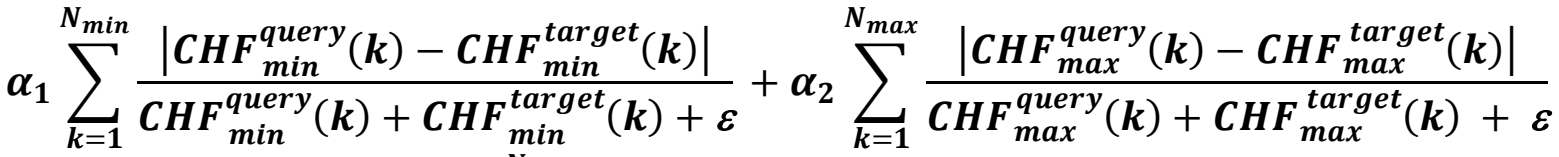

$$
\begin{aligned}
& +\alpha_{3} \sum_{k=1}^{N_{b}} \frac{\left|B P F^{\text {query }}(k)-B F^{\text {target }}(k)\right|}{\operatorname{BPFery}_{(k)+B P F^{\text {target }}(k)+\varepsilon}}
\end{aligned}
$$

where $\alpha 1, \alpha 2$ and $\alpha 3$ denote the similarity weighting constants representing the percentage contribution of the CHF and BPF utilized in the proposed image retrieval process. When a constant is set at 1 , meaning the corresponding feature is employed in the distance computation, whereas the value 0 means the feature is disable.

\subsection{Relative distance measure}

Here to compare the similarity of two images based on BCCM and $\mathrm{BPH}$, relative distance measure is used. Let $\mathrm{BCCM}_{\mathrm{p}}, \mathrm{BPH}_{\mathrm{p}}$ and $\mathrm{BCCM}_{\mathrm{q}}, \mathrm{BPH}_{\mathrm{q}}$ be the $\mathrm{BCCM}$ and $\mathrm{BPH}$ of images $\mathrm{p}$ and q respectively. The similarity of the images is measured as the distances between the BCCM's and BPH's $d(p, q)$ and is calculated as follows $[3,12-13]$ :

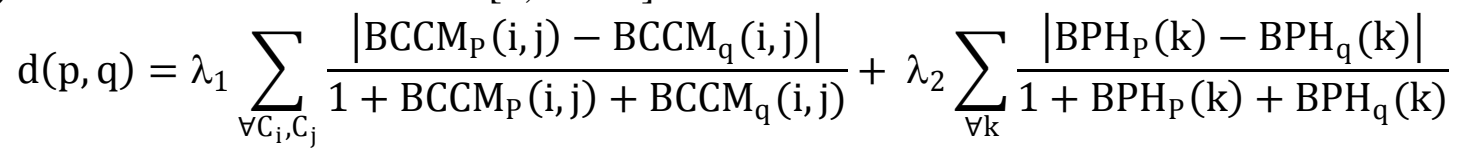

where $\lambda_{1}$ and $\lambda_{2}$ are weighting constants. Note that the two descriptors, BCCM and BPH belong to different modalities. It is popular to combine features of different modalities in image retrieval.

\section{PERFORMANCE EVALUATION FOR CBIR}

The effectiveness of the retrieval system technique is measured with the precision, recall, Average Precision Rate (APR) and Average Retrieval Rate (ARR) values [32]. These values indicate the percentage of relevant image returned by a CBIR system with a specific number of retrieved images. A higher value on precision, recall, APR and ARR indicate the higher retrieval rate or better retrieval performance.

\subsection{Precision}

Precision [4], is defined as the ratio of the number of retrieved relevant images to the total number of retrieved images.

Precision $=$ Number of relevant images retrieved Total number of images retrieved

\subsection{Recall}

Recall [4], is defined as the ratio of the number of retrieved relevant images to the total number of relevant images in the whole database.

\section{Recall $=\quad$ Number of relevant images retrieved Total number of relevant images in the database}

\subsection{Average Precision Rate (APR) and Average Retrieval Rate (ARR)}

The APR and ARR investigate how many relevant images returned by image retrieval system under a fix number of retrieved images. The higher value of APR and ARR exhibits the better image retrieval performance

The APR and ARR [14] are formally defined as

$$
\mathrm{APR}=\frac{1}{\mathrm{~N}_{\mathrm{t}} \mathrm{L}} \sum_{\mathrm{q}=1}^{\mathrm{N}_{\mathrm{t}}} \mathrm{n}_{\mathrm{q}}(\mathrm{L})
$$


where,

$$
\operatorname{ARR}=\frac{1}{\mathrm{~N}_{\mathrm{t}} \mathrm{N}_{\mathrm{R}}} \sum_{\mathrm{q}=1}^{\mathrm{N}_{\mathrm{t}}} \mathrm{n}_{\mathrm{q}}\left(\mathrm{N}_{\mathrm{R}}\right)
$$

$L \quad$ number of retrieved images

$\mathrm{N}_{\mathrm{t}} \quad$ total number of images in database

$\mathrm{N}_{\mathrm{R}} \quad$ number of relevant images on each class

q query image

$\mathrm{n}_{\mathrm{q}} \quad$ number of relevant images against a query image $\mathrm{q}$

$\mathrm{n}_{\mathrm{q}}(\mathrm{L})$ number of relevant images among $\mathrm{L}$ retrieved images

$\mathrm{n}_{\mathrm{q}}\left(\mathrm{N}_{\mathrm{R}}\right)$ number of relevant images in database images

\subsection{Normalised Modified Retrieval Rank (NMRR) and Average NMRR (ANMRR)}

The retrieval results can be measured by NMRR. It is not only provides how much of the correct items are retrieved, but also how highly they are ranked among the retrieved items. The NMRR is in the range of [0 1] and the smaller values represent a better retrieval performance.

The standard performance measure $N M R R$ is defined as

where,

$$
\operatorname{NMRR}(\mathrm{n})=\frac{\left(\sum_{k=1}^{N G(n)} \frac{\operatorname{Rank}(k)}{N G(n)}\right)-0.5-\frac{N G(n)}{2}}{K+0.5-0.5 * N G(n)}
$$

$N G \quad$ number of ground truth items marked as similar to the query item

$\operatorname{Rank}(k)$ ranking of the ground truth items by the retrieval algorithm

$K \quad$ equals to $\min (4 * N G(q), 2 * G T M)$

GTM the maximum of $N G(q)$ for the all queries.

$A N M R R$ [12] is defined as the Average $N M R R$ over a range of queries. To obtain the average of several queries the $A N M R R$ uses this expression where $N Q$ is the number of queries done.

$$
\operatorname{ANMRR}(\mathrm{q})=\frac{1}{\mathrm{NQ}} \sum_{\mathrm{q}=1}^{\mathrm{NQ}} \operatorname{NMRR}(\mathrm{q})
$$

\section{BTC BASED CBIR}

Several extensions of BTC compression techniques used for CBIR are discussed below with suitable examples and block diagrams. The methodologies taken for this survey of BTC based CBIR are: Block Truncation Coding (BTC), Error Diffusion BTC (EDBTC), Ordered Dither Block Truncation Coding (ODBTC), Dot diffusion BTC (DDBTC) .

\subsection{BLOCK TRUNCATION CODING (BTC)}

In Ref. [1], E.J. Delp and O.R. Mitchell (1979) proposed Block Truncation Coding (BTC) in 1979 for grayscale image compression. BTC has been considered as a highly efficient lossy type image compression technique for digital gray-level images. BTC has many advantages such as simple, efficient and low computation complexity. BTC preserves the standard mean and standard deviation of each image block. The BTC based schemes are suitable for image retrieval requiring fast execution. BTC produces two quantizers (high and low quantizers) and a bitmap image at the end of the encoding process is used for generating feature vectors.

In BTC, the original image is divided into fixed-size non overlapping blocks of size $M \times N$ with the size of $\mathrm{m}$ pixels in each block. To avoid the edge blurring and blocking effect, the block size chosen is usually small. The mean value $(\overline{\mathrm{x}})$ and standard deviation $(\sigma)$ are calculated for $x^{\text {th }}$ block as below.

$$
\overline{\mathrm{x}}=\frac{1}{\mathrm{~m}} \sum_{\mathrm{i}=1}^{\mathrm{m}} \mathrm{x}_{\mathrm{i}} \quad \overline{\mathrm{x}^{2}}=\frac{1}{\mathrm{~m}} \sum_{\mathrm{i}=1}^{\mathrm{m}} \mathrm{x}_{\mathrm{i}}^{2} \quad \sigma=\sqrt{\overline{\mathrm{x}^{2}}-\overline{\mathrm{x}}^{2}}
$$


The first and second order moments $\left(\overline{\mathrm{x}}, \overline{\mathrm{x}^{2}}\right)$ are preserved in the compression. Then a two-level quantization is performed (Let, $\mathrm{X}_{\mathrm{L}}=$ lower quantizer and $\mathrm{X}_{\mathrm{H}}=$ higher quantizer) to replace the pixel below mean value (by $X_{L}$ ) and above mean value (by $X_{H}$ ).

$$
X_{L}=\bar{x}-\sigma \sqrt{\frac{q}{m-q}} \quad X_{H}=\bar{x}+\sigma \sqrt{\frac{m-q}{q}}
$$

where $\mathrm{q}$ is the number of pixels greater than the mean value. Finally it produces two $\left(\mathrm{X}_{\mathrm{L}}\right.$ and $\left.\mathrm{X}_{\mathrm{H}}\right)$ quantizers, and one Bitmap image. At decoding stage, the pixel positions of each block, where the corresponding bitmap image $(\mathrm{O})$ has a value of 1 is replaced by one mean pixel value $\left(\mathrm{X}_{\mathrm{H}}\right)$ and those pixel positions where the corresponding bitmap has a value of 0 is replaced by another mean pixel $\operatorname{value}\left(\mathrm{X}_{\mathrm{L}}\right)$.

The reconstructed (decoding) image $\mathrm{O}$ is simultaneously generated at the same time with the bitmap image generation. The intermediate value $\mathrm{o}(\mathrm{x}, \mathrm{y})$ can be calculated as

$$
o(x, y)= \begin{cases}x_{L}, & \text { if } b(x, y)=0 \\ x_{H}, & \text { if } b(x, y)=1\end{cases}
$$

An example of BTC encoding and decoding shown below.

Original Image Pixels of a
\begin{tabular}{|r|r|r|r|} 
Block (A) \\
\hline 5 & 9 & 7 & 9 \\
\hline 7 & 11 & 18 & 7 \\
\hline 2 & 6 & 11 & 6 \\
\hline 5 & 5 & 8 & 10 \\
\hline
\end{tabular}

Bitmap Image of a Block (B)

Reconstructed Image of a Block (O)

$$
\begin{gathered}
m=16 \\
\bar{x}=7.88 \\
\overline{x^{2}}=74.38 \\
\sigma=3.52
\end{gathered}
$$

\begin{tabular}{|l|l|l|l|}
\hline 0 & 1 & 0 & 1 \\
\hline 0 & 1 & 1 & 0 \\
\hline 0 & 0 & 1 & 0 \\
\hline 0 & 0 & 1 & 1 \\
\hline
\end{tabular}

\begin{tabular}{|r|r|r|r|}
\hline 5 & 12 & 5 & 12 \\
\hline 5 & 12 & 12 & 5 \\
\hline 5 & 5 & 12 & 5 \\
\hline 5 & 5 & 12 & 12 \\
\hline
\end{tabular}

$$
\begin{gathered}
q=7 \\
X_{L}=4.77 \cong 5 \\
X_{H}=11.86 \cong 12
\end{gathered}
$$$$
o(x, y)= \begin{cases}X_{L}, & \text { if } b(x, y)=0 \\ X_{H}, & \text { if } b(x, y)=1\end{cases}
$$

\subsubsection{BTC based CBIR}

In Ref. [3], Guoping Qiu (2003) presented a method for using a well known image coding technique to achieve both image coding and content based image retrieval in the compressed domain. From the BTC compressed stream (without performing decoding), two image content description features derived directly. One termed the Block Colour Cooccurrence Matrix (BCCM), characterizes the statistics of the color correlation within the blocks. And the other Block Pattern Histogram (BPH), characterizes the statistics of the spatial patterns of the blocks. He used BCCM and BPH to compute the similarity measures of images in the retrieval of images in database applications. One significant advantage of this method is that it achieves coding and retrieval simultaneously. This method tested using 235 number of 512 x 512 pixel, 24 bits/pixel true colour texture images from MIT Media Lab's VisTex collection \& 20,000 photographic colour images from the commercially available CorelPhoto Collections. The performance was measured in terms of the retrieval rate (RR).

In Ref. [16],[17], Dr.H.B.Kekre, Sudeep D. Thepade $(2008,2009)$ proposed the block truncation coding (BTC) based feature for CBIR methods using various color features of image. Basically red, green and blue planes of image together considered to compute feature vector for CBIR. In [16], BTC based CBIR boosted for Kekre's LUV color space and in [17], YCbCr Color Space are considered. The two mean colors, Upper Mean and Lower Mean form a feature vector of the image. For all images stored in the database these feature vectors are computed and stored in feature vector table. This proposed method used Direct Euclidean Distance as a similarity measure to compute the similarity measures of images. This method tested on the 1000 number of Wang images with variable sizes spread across 11 categories of human being, animals, natural scenery and manmade things. To compare the techniques and to check their Performance, the precision and recall used. 
In Ref. [18], Dr.H.B.Kekre (2010) proposed improved CBIR using Multileveled Block Truncation Coding. Here the color averaging methods used are BTC Level-1, BTC Level-2, BTC Level-3. So the feature vector size per image is greatly reduced by using mean of each plane and find out the threshold value then divide each plane using threshold value.

In Ref. [19], Dr.H.B.Kekre, Sudeep D. Thepade (2012) proposed the block truncation coding (BTC) with assorted color spaces for finding feature vector in Content Based Image Classification (CBIC) techniques. Overall six color spaces have been explored which includes RGB color space for applying BTC.

\subsection{ERROR DIFFUSION BTC (EDBTC)}

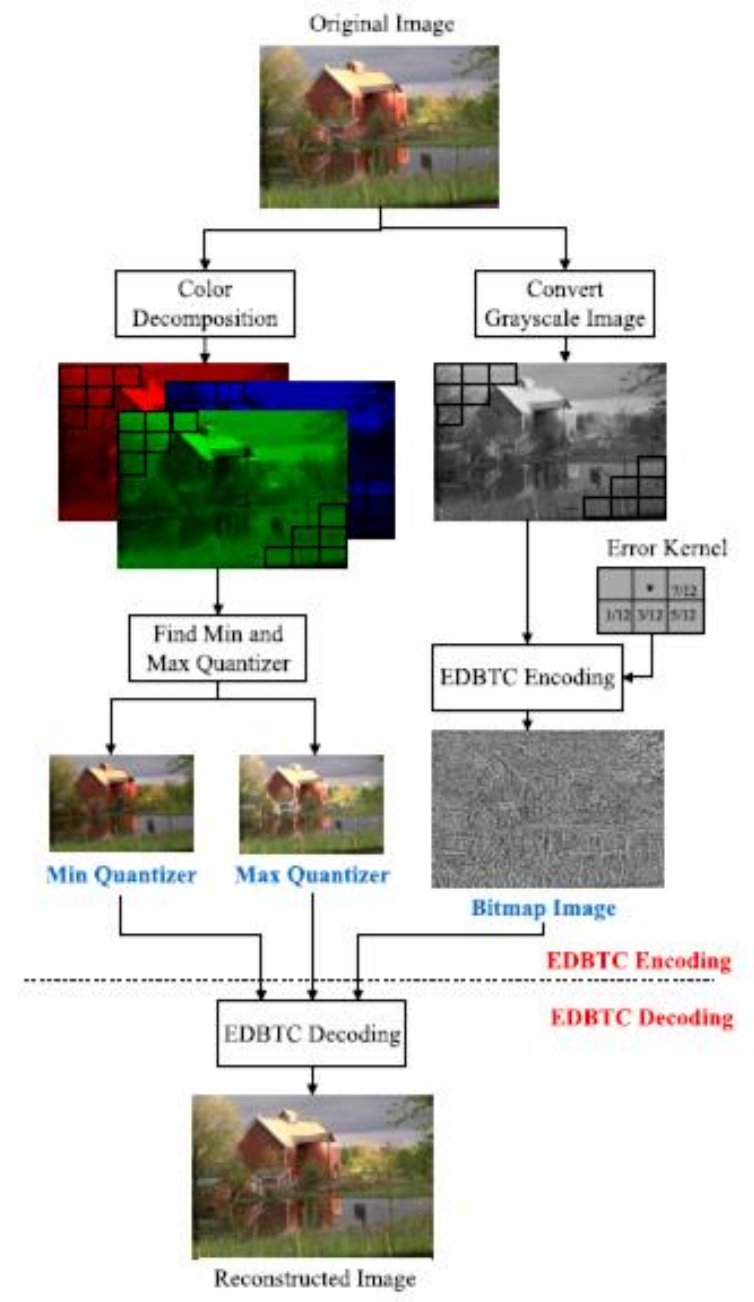

Fig 7. Schematic Diagram of the EDBTC encoding for a color image

The EDBTC [5],[10],[15],[22] is an improved version of BTC which is an efficient image compression technique. Error diffusion is a method that provides better visual quality of image. Error diffusion based BTC does this while also compressing the image. In this method, the inherent dithering property of error diffusion is to deal with the problem of false contour. Similar to the BTC scheme, EDBTC produces two quantizer and bitmap image for reducing the storage requirement. The EDBTC bitmap image is constructed by considering the quantized error which diffuses to the nearby pixels to compensate the overall brightness. EDBTC use the error kernel to generate the representative bitmap image. Several error kernels can be used to perform the diffusion operation, such as Jarvis error kernel, Burkers, Floyd-Steinberg, Sierra, Stucki and Stevenson. Different error kernel yield different halftoning pattern. Error diffusion strategy effectively removes the annoying blocking effect and false contour, while maintaining the low computational complexity. 


$$
\left[\begin{array}{ccc}
0 & 0 & 0 \\
0 & 0 & \frac{7}{12} \\
\frac{1}{12} & \frac{3}{12} & \frac{5}{12}
\end{array}\right]
$$

Floyd

$$
\left[\begin{array}{ccccc}
0 & 0 & 0 & 0 & 0 \\
0 & 0 & 0 & 0 & 0 \\
0 & 0 & 0 & \frac{7}{48} & \frac{5}{48} \\
\frac{3}{48} & \frac{5}{48} & \frac{7}{48} & \frac{5}{48} & \frac{3}{48} \\
\frac{1}{48} & \frac{3}{48} & \frac{5}{48} & \frac{3}{48} & \frac{1}{48}
\end{array}\right]
$$

Jarvis

$$
\left[\begin{array}{ccccc}
0 & 0 & 0 & 0 & 0 \\
0 & 0 & 0 & 0 & 0 \\
0 & 0 & 0 & \frac{8}{42} & \frac{4}{42} \\
\frac{2}{42} & \frac{4}{42} & \frac{8}{42} & \frac{4}{42} & \frac{2}{42} \\
\frac{1}{42} & \frac{2}{42} & \frac{4}{42} & \frac{2}{42} & \frac{1}{42}
\end{array}\right]
$$

Stucki

\section{Fig 8. Error Kernels of the EDBTC scheme}

The Fig.7 show the EDBTC encoding for a color image and Fig.8 shows some well known error kernels (error weights) of the EDBTC scheme.

\subsubsection{EDBTC based CBIR}

In Ref. [13],[21], J. M. Guo et.al., (2015) presented a new approach to index color images using the features extracted from the Error Diffusion Block Truncation Coding (EDBTC). The EDBTC produced two color quantizers and a bitmap image, which were further processed using vector quantization (VQ) to generate the image feature descriptor. The author introduced the color histogram feature (CHF) and bit pattern histogram feature (BHF). The CHF computed from the VQindexed color quantizer and BHF from VQ-indexed bitmap image. It measures the similarity between a query image and the target image in database. The distance computed from CHF and BHF used to measure the similarity between two images.

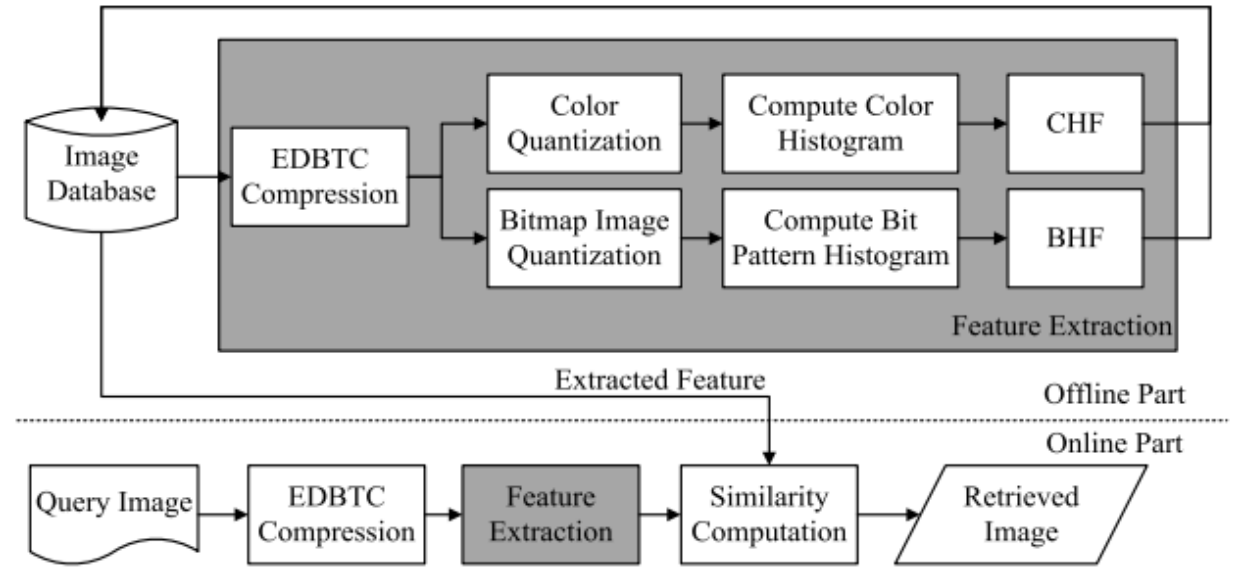

Fig 9. Block diagram of the image retrieval using EDBTC based indexing method

\subsection{ORDERED DITHER BTC (ODBTC)}

In Ref. [7],[8], J. M. Guo (2009, 2010) proposed an improved dithering based BTC algorithm called Ordered Dither Block Truncation Coding (ODBTC) for image compression. The ODBTC image compression is on its low complexity in generating bitmap image by including the Look-Up Table (LUT), and free of mathematical multiplication and division operations on the determination of the two extreme quantizers. The typical BTC requires additional computational time to derive the low and high mean values by preserving the first-order moment and second-order moment over each image block. But ODBTC identifies the minimum and maximum values of each image block as opposed to the former low and high mean values calculation, which can further reduce the processing time in the encoding stage. In addition, the ODBTC yields better reconstructed image quality by the extreme-value dithering effect while comparing with the typical BTC method. 


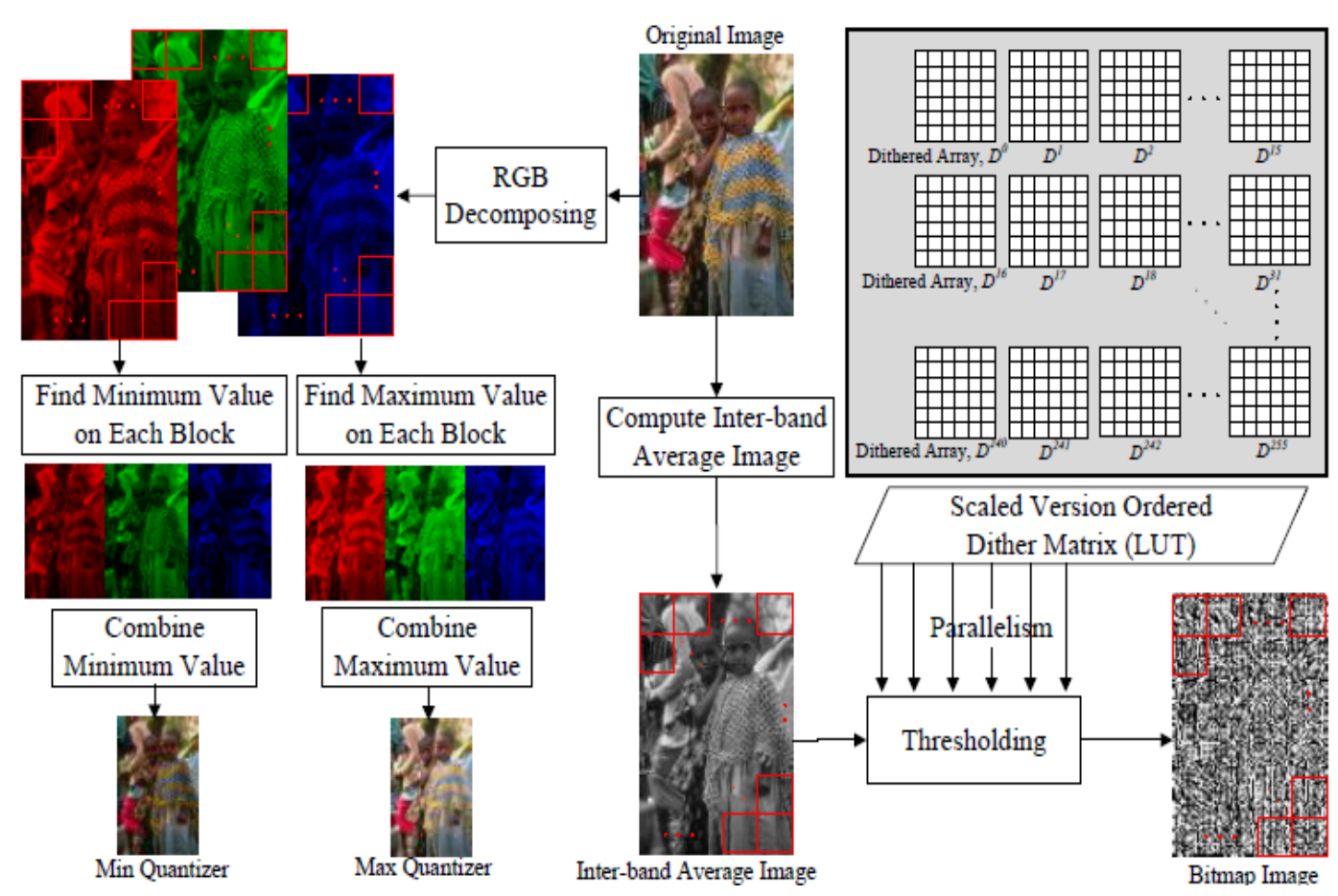

Fig 10. Schematic Diagram of the ODBTC encoding for a color image

\subsubsection{ODBTC based CBIR}

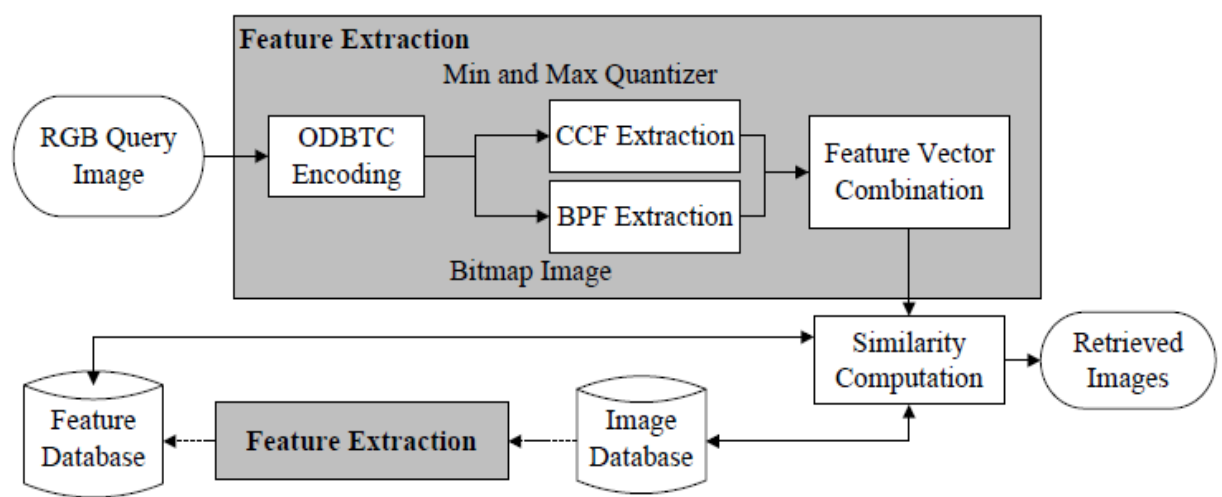

Fig 11. Block diagram of the image retrieval using ODBTC based indexing method

In Ref. [12], J. M. Guo et.al., (2015) proved that the ODBTC technique is well suited for CBIR system due to the low complexity and effective descriptor to index images. Two image features were proposed to index an image, namely, color co-occurrence feature (CCF) and bit pattern features (BPF), which are generated directly from the ODBTC encoded data streams without performing the decoding process. The CCF computed from the color quantizer and BPF derived from bitmap ODBTC by involving the visual codebook. The performance of proposed feature descriptor is tested for CBIR system using Corel, Brodatz-1856, Vistex-640, ALOT, Holidays, UKBench, Corel-DB, and Vistex-DB image databases. Four quantitative evaluations are used to examine the performance of proposed method, i.e., precision, recall, Average Retrieval Rate (ARR), and Average Normalized Modified Retrieval Rank (ANMRR).

\subsection{DOT DIFFUSION BTC (DDBTC)}

In Ref.[2],[7],[9],[11], J.M.Guo, et.al (2014) proposed Dot Diffusion BTC and proved it is superior to the earlier BTC in terms of various objective image quality assessment methods as well as processing efficiency. It improves the reconstructed image quality by reducing the blocking effect and false contour problem while maintaining the low computation complexity. This method provide 
excellent processing efficiency by exploiting the nature parallelism advantage of the dot diffusion, and excellent image quality also offered through co-optimizing the class matrix and diffused matrix of the dot diffusion.

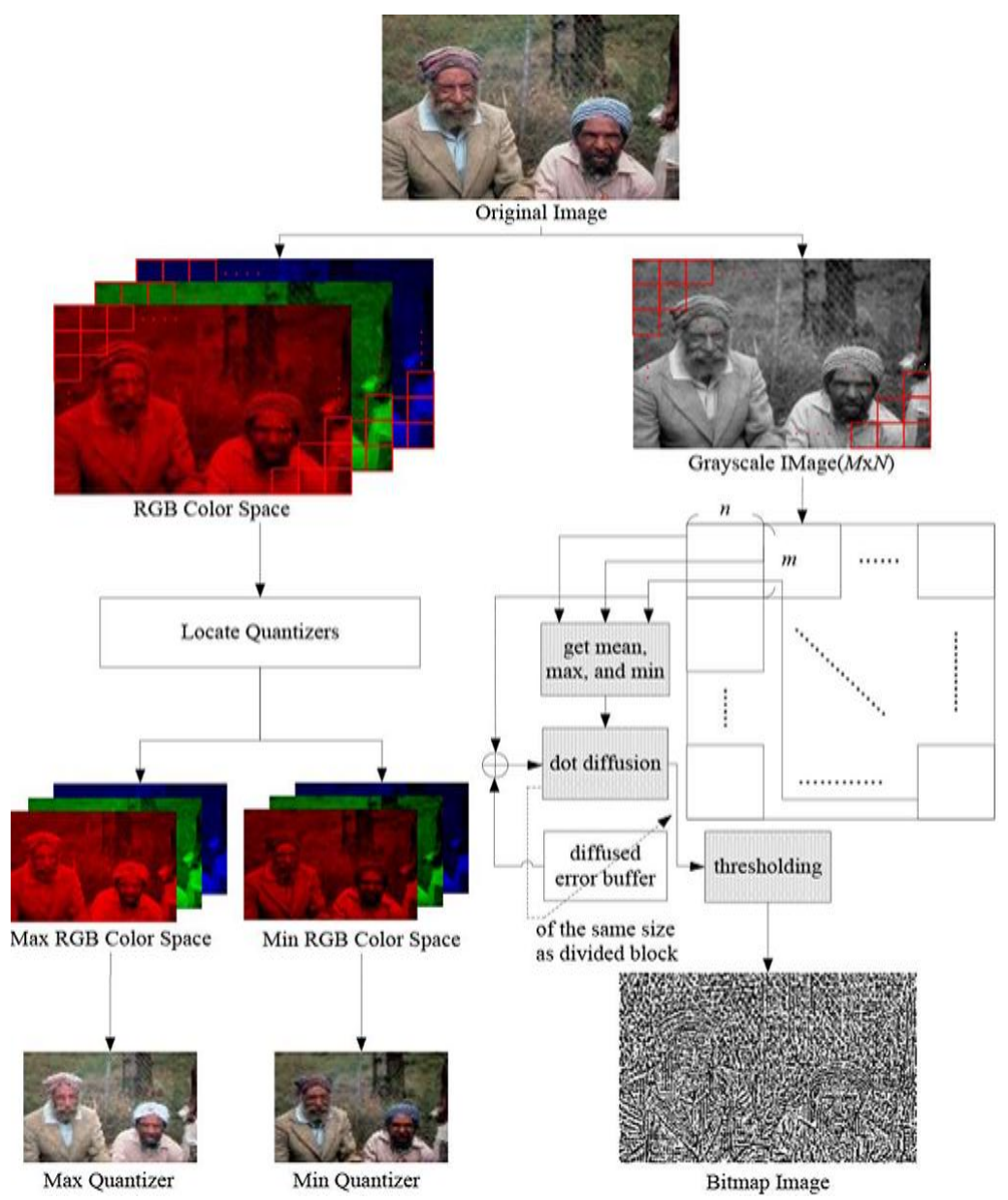

Fig 12. Schematic diagram of DDBTC encoding for color image.

\subsubsection{DDBTC based CBIR}

In Ref.[14] J. M. Guo, et.al (2015) used the Dot Diffused Block Truncation Coding (DDBTC) compressed data stream for CBIR system. The image feature descriptor is simply constructed from two DDBTC representative color quantizers and its corresponding bitmap image. The color histogram feature (CHF) derived from two color quantizers represents the color distribution and image contrast, while the bit pattern feature (BPF) constructed from the bitmap image characterizes the image edges and textural information. The similarity distance between the query and target images stored in database is computed based on their CHF and BPF to measure their similarity. The similarity between the query image and target image in database is computed using $L_{1}, L_{2}, \chi^{2}, F u$ and Modified Canberra distances. The performance is examined in terms of APR and ARR. The image databases used in this experiment are in the RGB color space as well as in the grayscale format. The image databases, namely Corel 1000, Brodatz-1856, Vistex-640, ALOT, Holidays, UKBench, CorelDB, and Vistex-DB are used. The DDBTC method compresses an image efficiently, and at the same time, its corresponding compressed data stream can provide an effective feature descriptor for performing image retrieval and classification. 

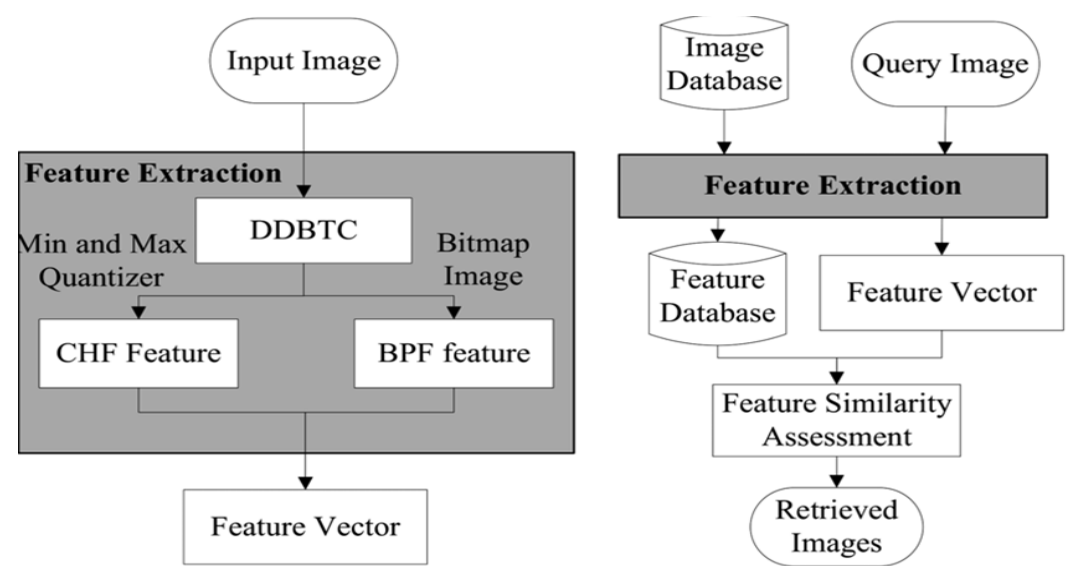

Fig 13. Block diagram of the image retrieval using DDBTC based indexing method

The Table 1 shows the summary of BTC based CBIR methods discussed in the literature survey.

Table 1. Comparison of various BTC based CBIR methods

\begin{tabular}{|c|c|c|c|c|c|c|c|}
\hline $\begin{array}{c}\text { Referred } \\
\text { Paper }\end{array}$ & Year & $\begin{array}{c}\text { BTC } \\
\text { Compression } \\
\text { Method Used }\end{array}$ & $\begin{array}{l}\text { Extracted } \\
\text { Features }\end{array}$ & $\begin{array}{l}\text { Color } \\
\text { Space } \\
\text { Used }\end{array}$ & Data Set Used & $\begin{array}{c}\text { Similarity } \\
\text { Measurement } \\
\text { Used }\end{array}$ & $\begin{array}{l}\text { Performance } \\
\text { Evaluation } \\
\text { Metric Used }\end{array}$ \\
\hline $\begin{array}{c}\text { Guoping } \\
\text { Qiu [3] }\end{array}$ & $\begin{array}{c}\text { Jan } \\
2003\end{array}$ & $\begin{array}{c}\text { Typical } \\
\text { BTC }\end{array}$ & $\begin{array}{c}\text { Block Colour Co- } \\
\text { occurrence Matrix } \\
\text { (BCCM) and } \\
\text { Block Pattern } \\
\text { Histogram (BPH) }\end{array}$ & RGB & $\begin{array}{l}\text { MIT Media Lab's } \\
\text { VisTex collection } \\
\text { \& photographic } \\
\text { images from } \\
\text { CorelPhoto } \\
\text { Collections }\end{array}$ & $\begin{array}{l}\text { Relative } \\
\text { distance } \\
\text { measure }\end{array}$ & $\begin{array}{c}\text { Retrieval } \\
\text { Rate(RR) } \\
\text { (this measure } \\
\text { is also known as } \\
\text { precision) }\end{array}$ \\
\hline $\begin{array}{l}\text { J. M. Guo } \\
\text { et.al., [12] }\end{array}$ & $\begin{array}{l}\text { Mar } \\
2015\end{array}$ & ODBTC & $\begin{array}{c}\text { Color Co- } \\
\text { occurrence Feature } \\
(\mathrm{CCF}) \text { and } \\
\text { Bit pattern } \\
\text { Features (BPF) }\end{array}$ & RGB & $\begin{array}{c}\text { Corel, Brodatz- } \\
\text { 1856, Vistex-640, } \\
\text { ALOT, } \\
\text { Holidays, } \\
\text { UKBench, Corel- } \\
\text { DB, and Vistex- } \\
\text { DB }\end{array}$ & $\begin{array}{l}\text { Relative } \\
\text { distance } \\
\text { measure }\end{array}$ & $\begin{array}{c}\text { precision, recall, } \\
\text { Average Retrieval } \\
\text { Rate (ARR), } \\
\text { and Average } \\
\text { Normalized } \\
\text { Modified } \\
\text { Retrieval Rank } \\
\text { (ANMRR) }\end{array}$ \\
\hline $\begin{array}{l}\text { J. M. Guo } \\
\text { et.al., [13] }\end{array}$ & $\begin{array}{l}\text { Mar } \\
2015\end{array}$ & EDBTC & $\begin{array}{c}\text { Color } \\
\text { Histogram Feature } \\
\text { (CHF) and Bit } \\
\text { Pattern Histogram } \\
\text { Feature } \\
\text { (BHF) }\end{array}$ & RGB & $\begin{array}{l}\text { Corel photo } \\
\text { collections }\end{array}$ & $\begin{array}{l}\text { Relative } \\
\text { distance } \\
\text { measure }\end{array}$ & $\begin{array}{l}\text { precision, recall, } \\
\text { and average } \\
\text { retrieval } \\
\text { rate (ARR) values }\end{array}$ \\
\hline $\begin{array}{l}\text { J. M. Guo } \\
\text { et.al., [14] }\end{array}$ & $\begin{array}{c}\text { Sep } \\
2015\end{array}$ & DDBTC & $\begin{array}{c}\text { Color } \\
\text { Histogram Feature } \\
(\mathrm{CHF}) \\
\text { and Bit Pattern } \\
\text { Feature (BPF) }\end{array}$ & $\begin{array}{c}\text { RGB } \\
\& \\
\text { Graysca } \\
\text { le }\end{array}$ & $\begin{array}{c}\text { Corel 1000, } \\
\text { Brodatz-1856, } \\
\text { Vistex-640, } \\
\text { ALOT, } \\
\text { Holidays, } \\
\text { UKBench, Corel- } \\
\text { DB, and Vistex- } \\
\text { DB }\end{array}$ & $\begin{array}{l}\mathrm{L} 1, \mathrm{~L} 2, \chi 2, \\
\text { Fu, and } \\
\text { Modified } \\
\text { Canberra }\end{array}$ & $\begin{array}{l}\text { Average } \\
\text { Precision Rate } \\
\text { (APR) and } \\
\text { Average Recall } \\
\text { Value (ARR) }\end{array}$ \\
\hline
\end{tabular}

\section{CONCLUSION}

In this literature survey, the content based image retrieval techniques using Block Truncation Coding based compression schemes for color image has been scrutinized. Four techniques were selected for compression specifically, the typical Block Truncation Coding (BTC), Error Diffusion Block Truncation Coding (EDBTC), Ordered Dither Block Truncation Coding (ODBTC) and Dot Diffusion Block Truncation Coding (DDBTC). The BTC based techniques are suitable for image retrieval requiring fast execution since their simplicity, low computational burden, fault tolerance, the relatively high compression efficiency and good image quality of the decoded image. This survey starts with a brief introduction about CBIR basic concepts and its usage. In this survey find out that 
BTC based CBIR not only applied for gray scale image it can be extended for color image. For future study, these image retrieval schemes can be applied to Satellite Image Retrieval and Video Retrieval. New features can be added by extracting the BTC based compressed images, not only $\mathrm{CCF} / \mathrm{CHF}$ and $\mathrm{BPF} / \mathrm{BHF}$, to enhance the retrieval performance. Since similarity distance is the heart of image retrieval system, an effective and suitable similarity distance can be applied to improve the overall performance. The user relevance feedback scheme can be added to retrieve more meaningful results.

\section{REFERENCES}

[1] E.J. Delp, O.R. Mitchell, "Image compression using block truncation coding”, IEEE Transactions on Communications COMM-27 (September 1979) 1335-1342.

[2] D. E. Knuth, "Digital halftones by dot diffusion", ACM Trans. Graph., Vol. 6, No. 4, pp. 245 273, Oct. 1987.

[3] Guoping Qiu, "Color Image Indexing Using BTC", IEEE Transactions on Image Processing, Vol.12, No.1, pp.93-101, Jan. 2003

[4] Y. Liu et al., "A survey of content based image retrieval with high-level semantics", Journal of Pattern Recognition, Vol. 40, pp. 262-282, Nov. 2007.

[5] J. M. Guo, "Improved block truncation coding using modified error diffusion", Electron. Lett., Vol. 44, No. 7, Mar. 2008, pp. 462-464.

[6] J. M. Guo and M. F. Wu, "Improved Block Truncation Coding Based on the Void-and-Cluster Dithering Approach,” IEEE Trans. Image Processing, vol. 18, no. 1, pp. 211-213, Jan. 2009.

[7] J. M. Guo and Yun-Fu Liu," Improved Dot Diffusion by Diffused Matrix and Class Matrix CoOptimization”, IEEE Trans. On Image Processing,, VoL. 18, No. 8, Aug. 2009

[8] J. M. Guo, "High efficiency ordered dither block truncation coding with dither array LUT and its scalable coding application,” Digit. Signal Process., Vol. 20, No. 1, pp. 97-110, Jan. 2010.

[9] J. M. Guo and Y. F. Liu, "Improved block truncation coding using optimized dot diffusion," in Proc. IEEE Int. Symp. Circuits Syst., May-Jun. 2010, pp. 2634-2637.

[10]J. M. Guo and Y.-F. Liu, "Joint compression/watermarking scheme using majority-parity guidance and halftoning-based block truncation coding," IEEE Trans. Image Process., Vol. 19, No. 8, pp. 2056-2069, Aug. 2010.

[11] J. M. Guo and Y. F. Liu, "Improved Block Truncation Coding Using Optimized Dot Diffusion", IEEE Trans. On Image Processing, Vol. 23, No. 3, Mar. 2014

[12] J. M. Guo, and H. Prasetyo,"Content-Based Image Retrieval Using Features Extracted From Halftoning-Based Block Truncation Coding”, IEEE Transaction on Image Processing , Vol. 24 , No. 3, Mar. 2015.

[13] J. M. Guo, H. Prasetyo, and Jen-Ho Chen, "Content-Based Image Retrieval Using Error Diffusion Block Truncation Coding Features", IEEE Transactions On Circuits And Systems For Video Technology, Vol. 25, No. 3, Mar. 2015.

[14]J. M. Guo, H. Prasetyo, and N. J. Wang, "Effective image retrieval system using dot-diffused block truncation coding features," IEEE Trans. on Multimedia, Vol. 17, No. 9, Sep. 2015.

[15] K. Knox, "Evolution of Error Diffusion", J. Electonic Imaging, Vol. 8, No. 4, pp 422-429, 1999.

[16]Dr.H.B.Kekre, Sudeep D. Thepade, "Boosting Block Truncation Coding using Kekre's LUV Color Space for Image Retrieval", WASET Int. Journal of Electrical, Computer and System Engineering (IJECSE), Vol. 2, No. 3, pp. 172-180, Summer 2008.

[17] Dr.H.B.Kekre, Sudeep D. Thepade, "Color Based Image Retrieval using Amendment Block Truncation Coding with YCbCr Color Space", International Journal on Imaging (IJI), Vol. 2, No. A09, Autumn 2009, pp. 2-14. 
[18]Dr. H.B.Kekre et al. "Improved CBIR using Multileveled Block Truncation Coding", International Journal on Computer Science and Engineering(IJCSE) Vol. 02, No. 07, 2010, 2471 2476

[19] Dr.H.B.Kekre, Sudeep D. Thepade," Image Classification using Block Truncation Coding with Assorted Color Spaces", International Journal of Computer Applications (0975 - 8887) Vol. 44, No.6, April 2012

[20]F. Long, H. J. Zhang, D. D. Feng (2003) , 'Fundamentals of content - based image retrieval', in: D. D. Feng, W. C. Siu, H. J .Zhang(Eds.), Multimedia Information Retrieval and ManagementTechnological Fundamentals and Applications, Springer, pp.1-27.

[21] J. M. Guo and H. Prasetyo "Performance Evaluation of Error Diffusion Block Truncation Coding Feature for Color Image Retrieval" Chapter 4, publically available in the link http://sciencegatepub.com/books/gcsr/gcsr_vol4/GCSR-Vol4-Ch4.pdf

[22]R. Ulichney, Digital Halftoning, The MIT Press, Cambridge, 1987.

[23] S.F. Chang, A. Eleftheriadis, R. McClintock "Next-generation content representation, creation, and searching for new-media applications in education", in IEEE Proceedings, Vol.86, 1998.

[24] J. Huang et al., "Image indexing using color correlogram," in Proc.CVPR97, 1997, pp. 762-768.

[25] M. R. Gahroudi and M. R. Sarshar, "Image retrieval based on texture and color method in BTCVQ compressed domain,” in Proc. Int. Symp. Signal Process. Appl., Feb. 2007, pp. 1-4.

[26] Corel Photo Collection Color Image Database. http://wang.ist.psu.edu/docs/related/.

[27]Z. M. Lu, and H. Burkhardt, "Colour image retrieval based on DCT-domain vector quantization index histograms," Electronics Letters, vol. 41, no. 17, 2005.

[28] P. Poursistani, H. Nezamabadi-pour, R. A. Moghadam, and M. Saeed, "Image indexing and retrieval in JPEG compressed domain based on vector quantization," Math. and Comp. Modeling, vol. 57, no. 5-6, pp. 1005-1017, 2013.

[29] Kim, Tae-Su \& Kim, Seung-Jin \& Lee, Kuhn-Il. (2005). Image Retrieval Based on Cooccurrence Matrix Using Block Classification Characteristics. 3767. 946-956. 10.1007/11581772_83.

[30] Jing-Ming Guo, Heri Prasetyo, Huai-Sheng Su. (2013). Image indexing using the color and bit pattern feature fusion. Journal of Visual Communication and Image Representation, Volume 24, Issue 8, November 2013, Pages 1360-1379.

[31]R. Sahaya Jeya Sutha, D.S. Mahendran, S. John Peter (2017). An Overview of Content Based Image Retrieval Techniques. International Journal of Advanced Research Trends in Engineering and Technology (IJARTET) Vol. 4, Special Issue 4, March 2017.

[32] P. Liu, J. M. Guo, C. Y. Wu and D. Cai, "Fusion of Deep Learning and Compressed Domain Features for Content-Based Image Retrieval," in IEEE Transactions on Image Processing, vol. 26, no. 12, pp. 5706-5717, Dec. 2017.

[33] Y. D. Chun, N. C. Kim, and I. H. Jang, Content -based image retrieval using multiresolution color and texture features, IEEE Trans. Multimedia, Vol. 10, No. 6, Pp. 1073 1084, Oct. 2008.

\section{ABOUT THE AUTHORS}

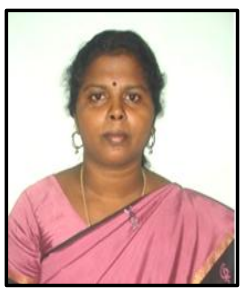

R. Sahaya Jeya Sutha received the B.Sc. (Computer Science), M.C.A. (Computer Applications) and M.Phil. (Computer Science) degrees in 1995, 2000 and 2006, respectively from the Manonmaniam Sundaranar University, Tirunelveli. She has qualified the Tamilnadu State Eligibility Test (SET) Lectureship in 2016. She is currently pursuing Ph.D. degree in Computer Applications at the Manonmaniam Sundaranar University, Tirunelveli. She is a life member of ISTE. Her research interests include image retrieval, video retrieval, data hiding and image compression. 


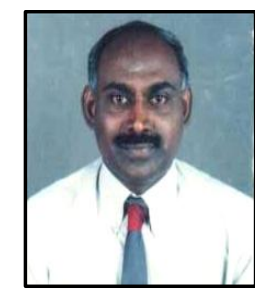

D.S. Mahendran is working as Associate Professor in Computer Science, Aditanar College, Tiruchendur. He received the M.Sc. Physics and PGDCA degrees from Madurai Kamaraj University and M.Phil. and Ph.D degrees in Computer Science from Alagappa University, Karaikudi. His research interest includes Computer algorithms, Ad-Hoc Networks, Network Security and Green Network.

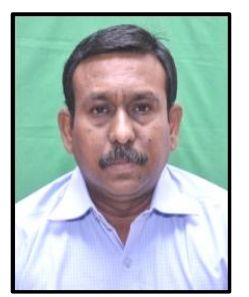

S. John Peter is working as Associate professor in Computer Science at St. Xavier's College, Palayamkottai, Tirunelveli. He received his M.Sc. and M.Phil. degrees in Computer Science from Bharadhidasan University, Tiruchirappalli. He also earned his Ph.D degree from Manonmaniam Sundaranar University, Tirunelveli. He has published many research papers on clustering algorithms in various conferences, national and international journals. 